\title{
CONVERGENCE OF SECOND-ORDER, ENTROPY STABLE METHODS FOR MULTI-DIMENSIONAL CONSERVATION LAWS
}

\author{
Neelabja Chatterjee ${ }^{1}$ And Ulrik Skre Fjordholm ${ }^{2}$
}

\begin{abstract}
High-order accurate, entropy stable numerical methods for hyperbolic conservation laws have attracted much interest over the last decade, but only a few rigorous convergence results are available, particularly in multiple space dimensions. In this paper we show how the entropy stability of one such method, which is semi-discrete in time, yields a (weak) bound on oscillations. Under the assumption of $L^{\infty}$-boundedness of the approximations we use compensated compactness to prove convergence to a weak solution satisfying at least one entropy condition.
\end{abstract}

2010 Mathematics Subject Classification. Primary: 35L65, 65M12; Secondary: 65M08.

The dates will be set by the publisher.

\section{INTRODUCTION}

Hyperbolic conservation laws appear in a large variety of applications, including gas dynamics, traffic modeling, multi-phase fluid flow problems, and more; see e.g. $[3,9,12,16]$. We consider a scalar, $d$-dimensional hyperbolic conservation law

$$
\begin{cases}\partial_{t} u+\nabla \cdot f(u)=0 & \forall(x, t) \in \mathbb{R}^{d} \times \mathbb{R}_{+} \\ u(x, 0)=u_{0}(x) & \forall x \in \mathbb{R}^{d}\end{cases}
$$

where $u=u(x, t): \mathbb{R}^{d} \times \mathbb{R}_{+} \rightarrow \mathbf{U}$ is the unknown conserved variable, taking values in some nonempty interval $\mathbf{U} \subset \mathbb{R}$, and the function $f=\left(f_{1}, \ldots, f_{d}\right): \mathbf{U} \rightarrow \mathbb{R}^{d}$ is the smooth (at least $C^{3}$ on $\mathbf{U}$ ) and possibly nonlinear flux function. For the sake of simplicity we will assume that $\mathbf{U}=\mathbb{R}$.

It is well-known that even if the initial datum $u_{0}(x)$ is arbitrarily smooth, the solutions of (1.1) may still be non-smooth $[3,9,12]$. Thus, it is fruitless to look for solutions of $(1.1)$ in the classical sense. Instead these solutions are sought in a weak sense. A function $u \in L^{\infty}\left(\mathbb{R}^{d} \times \mathbb{R}_{+}\right)$is said to be a weak solution of (1.1) if it is

Keywords and phrases: Multi-dimensional conservation laws; finite volume methods; TECNO scheme; entropy stability 1

Department of mathematics

University of Oslo, P.O. Box 1053, Blindern, 0316-Oslo, Norway

e-mail: nelabja12@gmail.com; neelabjc@math.uio.no

2

Department of mathematics

University of Oslo, P.O. Box 1053, Blindern, 0316-Oslo, Norway

e-mail: ulriksf@math.uio.no 
a distributional solution, i.e.

$$
\int_{\mathbb{R}^{d}} \int_{\mathbb{R}_{+}} \partial_{t} u \varphi+f(u) \cdot \nabla \varphi d x d t+\int_{\mathbb{R}^{d}} u_{0}(x) \varphi(x, 0) d x=0 \quad \forall \varphi \in C_{c}^{1}\left(\mathbb{R}^{d} \times[0, \infty)\right) .
$$

It is well known (see e.g. [3,9,12]) that weak solutions may be non-unique. Thus to single out a physically relevant solution, the notion of weak solution has to be supplemented with an additional admissibility criterion, namely entropy conditions. A pair of functions $\eta: \mathbb{R} \rightarrow \mathbb{R}, q: \mathbb{R} \rightarrow \mathbb{R}^{d}$ is an entropy pair for (1.1) if $\eta$, the entropy function, is convex and $q$, the entropy flux function, satisfies $q^{\prime}(u)=\eta^{\prime}(u) f^{\prime}(u)$. In particular, for every $k \in \mathbb{R}$ we have the well-known Kružkov entropy pair $\left(\eta_{k}, q_{k}\right)$ given by

$$
\eta_{k}(u):=|u-k|, \quad q_{k}(u):=\operatorname{sign}(u-k)(f(u)-f(k)),
$$

see [14]. Multiplying (1.1) by $\eta^{\prime}(u)$ and using the chain rule we have the following entropy conservation identity for smooth solutions of (1.1),

$$
\partial_{t} \eta(u)+\nabla \cdot q(u)=0 .
$$

Due to the possible non-smoothness of solutions of (1.1), the above derivation cannot be rigorously justified for weak solutions. Instead, motivated by the second law of thermodynamics, the entropy inequality

$$
\partial_{t} \eta(u)+\nabla \cdot q(u) \leqslant 0
$$

is imposed. As was shown by Kružkov [14], validity of this entropy condition for all Kružkov entropy pairs $\left(\eta_{k}, q_{k}\right)_{k \in \mathbb{R}}$ guarantees uniqueness and stability of solutions of (1.1).

\subsection{Numerical methods for conservation laws}

The nonlinear nature of the equation (1.1) and the fact that its solutions are irregular, can make the construction and analysis of numerical methods for (1.1) challenging. We outline here some of the available literature on this subject.

In order to converge to a weak solution satisfying the entropy condition (1.5), the numerical method needs to satisfy some discrete version of the entropy condition. Finite volume methods (to be discussed in subsection 2.1 in detail) with this property are called entropy stable. Harten, Hyman and Lax [10] showed that all monotone schemes for scalar conservation laws are entropy stable with respect to any entropy pair $(\eta, q)$. Osher [17] generalized this to a (presumably) larger class of schemes, the so-called E-schemes. Osher also showed that these E-schemes are at most first-order accurate. In his papers from 1984 [19] and from 1987 [20], Tadmor laid a general framework for constructing entropy stable schemes by first constructing entropy conservative methods schemes satisfying a discrete version of (1.4) - and then adding numerical diffusion to obtain entropy stability. As he proved in [20], entropy conservative schemes are generally second-order accurate; even higher-order entropy conservative schemes were constructed by Lefloch, Mercier and Rohde in [15]. However, the addition of numerical diffusion to any of these entropy conservative schemes, in the way suggested in [19,20], degrades the order of accuracy to 1. Following an alternate approach for higher order entropy stable methods, a discontinuous Galerkin method was designed in [2]. Convergence analysis of finite volume schemes on unstructured, quasi-uniform meshes for smooth solutions of first-order systems of hyperbolic balance laws in multiple space dimensions was carried out in [13].

By combining the high-order accurate entropy conservative schemes in $[15,20]$ with a judiciously chosen reconstruction method, Fjordholm, Mishra and Tadmor [4,7] constructed entropy stable methods with an arbitrarily high order of accuracy, the so-called TECNO schemes. By estimating the amount of entropy dissipated by the method (i.e., the amplitude of the left-hand side in (1.5)), the authors could derive a priori weak regularity bounds on the numerical solution, and these bounds, along with the assumption of $L^{\infty}$-boundedness, were sufficient to prove convergence of the method in the special case of $d=1$ space dimensions. 
To the best of our knowledge there is no available proof of convergence of a high-order accurate entropy stable method for a multi-dimensional conservation law. The purpose of the present paper is to prove convergence for a particular semi-discrete numerical method, namely the second-order TECNO scheme. A convergence proof for a fully discrete method would be similar but much more involved, since this would lead to much more delicate computations in deriving the a priori estimates that we require in our convergence argument. We refer to the earlier work [6] for entropy stable, fully discrete TECNO schemes. Finally, we note that our convergence proof requires an a priori $L^{\infty}$ bound on the numerical approximations; a proof of such a bound, while at the same time retaining the high order of accuracy, seems to be out of reach.

\section{ENTROPy STABLE NUMERICAL METHODS}

\subsection{Finite volume methods}

For the sake of notational simplicity we are going to consider the scalar conservation law (1.1) in the particular case of $d=2$ space dimensions, although we emphasize that the results in this paper are equally valid for any number of spatial dimensions $d$.

We write (1.1) in the case $d=2$ as

$$
\begin{cases}\partial_{t} u+\partial_{x} f^{x}(u)+\partial_{y} f^{y}(u)=0 & \forall(x, y, t) \in \mathbb{R}^{2} \times \mathbb{R}_{+} \\ u(x, y, 0)=u_{0}(x, y) & \forall(x, y) \in \mathbb{R}^{2} .\end{cases}
$$

Here and in the remainder we will denote the components of all vector-valued functions by $f=\left(f^{x}, f^{y}\right)$.

One of the most popular discretization frameworks is the finite volume method. The spatial domain $\mathbb{R}^{2}$ is partitioned into rectangles of the form $\mathcal{C}_{i, j}=\left[x_{i-1 / 2}, x_{i+1 / 2}\right) \times\left[y_{j-1 / 2}, y_{j+1 / 2}\right)$, where for the sake of simplicity we use uniform grid sizes $x_{i+1 / 2}-x_{i-1 / 2} \equiv \Delta x$ and $y_{j+1 / 2}-y_{j-1 / 2} \equiv \Delta y$. We denote the midpoint values as $x_{i}=\frac{x_{i-1 / 2}+x_{i+1 / 2}}{2}$ and $y_{j}=\frac{y_{j-1 / 2}+y_{j+1 / 2}}{2}$. For any quantity $\left(u_{i, j}\right)_{i, j \in \mathbb{Z}}$ defined on this grid, we define the jump and average operators

$$
\begin{array}{lrl}
\left\{\{u\}_{i+1 / 2, j}=\frac{u_{i, j}+u_{i+1, j}}{2}\right. & \llbracket u \rrbracket_{i+1 / 2, j}:=u_{i+1, j}-u_{i, j} \\
\left\{\{u\}_{i, j+1 / 2}=\frac{u_{i, j}+u_{i, j+1}}{2}\right. & \llbracket u \rrbracket_{i, j+1 / 2}:=u_{i, j+1}-u_{i, j} .
\end{array}
$$

We let $u_{i, j}(t)$ be an approximation of the average value of $u$ over the rectangles $\mathcal{C}_{i, j}$, that is,

$$
u_{i, j}(t) \approx \frac{1}{\Delta x \Delta y} \int_{\mathcal{C}_{i, j}} u(x, y, t) d(x, y) .
$$

The initial data is sampled as $u_{i, j}(0)=\frac{1}{\Delta x \Delta y} \int_{\mathcal{C}_{i, j}} u_{0}(x, y) d(x, y)$. A semi-discrete finite volume method for (2.1) can then be written in the generic form

$$
\frac{d}{d t} u_{i, j}(t)+\frac{F_{i+1 / 2, j}^{x}-F_{i-1 / 2, j}^{x}}{\Delta x}+\frac{F_{i, j+1 / 2}^{y}-F_{i, j-1 / 2}^{y}}{\Delta y}=0
$$

where the numerical flux function $F_{i+1 / 2, j}^{x}=F^{x}\left(u_{i, j}, u_{i+1, j}\right)$ is computed from an approximate solution of the Riemann problem at the interface $\left\{\left(x_{i+1 / 2}, y\right)\right\}_{y_{j-1 / 2} \leqslant y \leqslant y_{j+1 / 2}}$ and $F_{i, j+1 / 2}^{y}$ is computed analogously [9,12]. As for the flux function $f$, we will denote $F=\left(F^{x}, F^{y}\right)$. The computed solution generated by the scheme is given by $u^{\boldsymbol{\Delta}}(x, y, t)=\sum_{i, j} u_{i, j}(t) \chi_{\mathcal{C}_{i, j}}(x, y)$, where $\boldsymbol{\Delta}=(\Delta x, \Delta y)$ and $\chi_{\mathcal{C}}$ is the characteristic function for the rectangle $\mathcal{C}$. We say that the numerical flux function $F$ is consistent with $f$ if $F^{x}(u, u)=f^{x}(u)$ and $F^{y}(u, u)=f^{y}(u)$ for all $u \in \mathbb{R}$. We also say that a numerical flux $F$ is locally Lipschitz continuous if $F^{x}, F^{y}$ are locally Lipschitz continuous in each argument. 


\subsection{Entropy stable numerical methods}

In order for any limit $u=\lim _{\Delta \rightarrow 0} u^{\Delta}$ to satisfy the entropy condition (1.5), the numerical method (2.2) must satisfy some discrete form of the entropy condition. In this section we briefly review the theory of so-called entropy conservative and entropy stable schemes, and we define the TECNO schemes, which will be the subject of the rest of the paper.

Definition 2.1 (Entropy conservative methods). Let $(\eta, q)$ be an entropy pair. We say that the finite volume method (2.2) is entropy conservative (with respect to $(\eta, q)$ ) if computed solutions satisfy the discrete entropy equality

$$
\frac{d}{d t} \eta\left(u_{i, j}\right)+\frac{Q_{i+1 / 2, j}^{x}-Q_{i-1 / 2, j}^{x}}{\Delta x}+\frac{Q_{i, j+1 / 2}^{y}-Q_{i, j-1 / 2}^{y}}{\Delta y}=0
$$

where $Q_{i+1 / 2, j}^{x}=Q^{x}\left(u_{i, j}, u_{i+1, j}\right)$ and $Q_{i, j+1 / 2}^{y}=Q^{y}\left(u_{i, j}, u_{i, j+1}\right)$ are numerical entropy flux functions satisfying $Q^{x}(u, u)=q^{x}(u)$ and $Q^{y}(u, u)=q^{y}(u)$ for all $u \in \mathbb{R}$.

Definition 2.2 (Entropy stable methods). Let $(\eta, q)$ be an entropy pair. We say that the finite volume method (2.2) is entropy stable (with respect to $(\eta, q)$ ) if computed solutions satisfy the discrete entropy equality

$$
\frac{d}{d t} \eta\left(u_{i, j}\right)+\frac{Q_{i+1 / 2, j}^{x}-Q_{i-1 / 2, j}^{x}}{\Delta x}+\frac{Q_{i, j+1 / 2}^{y}-Q_{i, j-1 / 2}^{y}}{\Delta y} \leqslant 0
$$

where $Q_{i+1 / 2, j}^{x}=Q^{x}\left(u_{i, j}, u_{i+1, j}\right)$ and $Q_{i, j+1 / 2}^{y}=Q^{y}\left(u_{i, j}, u_{i, j+1}\right)$ are numerical entropy flux functions satisfying $Q^{x}(u, u)=q^{x}(u)$ and $Q^{y}(u, u)=q^{y}(u)$ for all $u \in \mathbb{R}$.

For an entropy pair $(\eta, q)$ the mapping $u \mapsto \eta^{\prime}(u)$ is of particular importance, and we denote this entropy variable by $v=v(u):=\eta^{\prime}(u)$. If $\eta$ is strictly convex, $\eta^{\prime \prime}(u)>0$, then the map $u \mapsto v(u)$ is strictly monotone increasing and hence is invertible. This inverse will be denoted by $u(v)$. Thus, the mapping $u \mapsto v$ induces a change of variables, in terms of which we can pose the conservation law (2.2) as

$$
\partial_{t} u(v)+\nabla \cdot f(u(v))=0 .
$$

We define also the entropy potential $\psi: \mathbb{R} \rightarrow \mathbb{R}^{d}$ defined by $\psi(u):=v(u) f(u)-q(u)$, whose name is given by the fact that $\partial_{v} \psi(u(v))=f(u(v))$.

A general approach to designing entropy conservative/stable schemes is as follows. Multiplying both sides of (2.2) by $v_{i, j}:=\eta^{\prime}\left(u_{i, j}\right)$ and using the chain rule we get

$$
\frac{d}{d t} \eta\left(u_{i, j}\right)+v_{i, j} \frac{F_{i+1 / 2, j}^{x}-F_{i-1 / 2, j}^{x}}{\Delta x}+v_{i, j} \frac{F_{i, j+1 / 2}^{y}-F_{i, j-1 / 2}^{y}}{\Delta y}=0 .
$$

Adding and subtracting terms yields

$$
\begin{aligned}
\frac{d}{d t} \eta\left(u_{i, j}\right)+ & \frac{Q_{i+1 / 2, j}^{x}-Q_{i-1 / 2, j}^{x}}{\Delta x}+\frac{Q_{i, j+1 / 2}^{y}-Q_{i, j-1 / 2}^{y}}{\Delta y} \\
= & \frac{r_{i+1 / 2, j}^{x}+r_{i-1 / 2, j}^{x}}{2 \Delta x}+\frac{r_{i, j+1 / 2}^{y}+r_{i, j-1 / 2}^{y}}{2 \Delta y}
\end{aligned}
$$

where

$$
\begin{aligned}
r_{i+1 / 2, j}^{x} & =\llbracket v \rrbracket_{i+1 / 2, j} F_{i+1 / 2, j}^{x}-\llbracket \psi^{x} \rrbracket_{i+1 / 2, j}, \\
r_{i, j+1 / 2}^{y} & =\llbracket v \rrbracket_{i, j+1 / 2} F_{i, j+1 / 2}^{y}-\llbracket \psi^{y} \rrbracket_{i, j+1 / 2} \\
Q_{i+1 / 2, j}^{x} & =\left\{\{v\}_{i+1 / 2, j} F_{i+1 / 2, j}^{x}-\left\{\left\{\psi^{x}\right\}_{i+1 / 2, j},\right.\right. \\
Q_{i, j+1 / 2}^{y} & =\left\{\{v\}_{i, j+1 / 2} F_{i, j+1 / 2}^{y}-\left\{\left\{\psi^{y}\right\}_{i, j+1 / 2} .\right.\right.
\end{aligned}
$$


It is straightforward to see that $Q^{x}, Q^{y}$ are consistent with $q$ in the sense of Definitions 2.1 and 2.2 , as long as $F^{x}, F^{y}$ are consistent with $f$. Thus, if $F^{x}, F^{y}$ are chosen such that either $r \equiv 0$ or $r \leqslant 0$, then the scheme $(2.2)$ is entropy conservative/stable. In particular, if $F^{x}, F^{y}$ are of the form

$$
F_{i+1 / 2, j}^{x}=\tilde{F}_{i+1 / 2, j}^{x}-D_{i+1 / 2, j}^{x} \llbracket v \rrbracket_{i+1 / 2, j}, \quad F_{i, j+1 / 2}^{y}=\tilde{F}_{i, j+1 / 2}^{y}-D_{i, j+1 / 2}^{y} \llbracket v \rrbracket_{i, j+1 / 2}
$$

for nonnegative coefficients $D^{x}, D^{y} \geqslant 0$ and numerical fluxes $\tilde{F}^{x}, \tilde{F}^{y}$ satisfying

$$
\llbracket v \rrbracket_{i+1 / 2, j} \tilde{F}_{i+1 / 2, j}^{x}=\llbracket \psi^{x} \rrbracket_{i+1 / 2, j}, \quad \llbracket v \rrbracket_{i, j+1 / 2} \tilde{F}_{i, j+1 / 2}^{y}=\llbracket \psi^{y} \rrbracket_{i, j+1 / 2}
$$

then the resulting scheme (2.2) is entropy stable. These observations were first made by Tadmor [19,20]; see also [21]. For fluxes of the form (2.8) we also get a precise expression for the amount of entropy dissipated in $(2.4)$ :

$$
\begin{aligned}
\frac{d}{d t} \eta\left(u_{i, j}\right) & +\frac{Q_{i+1 / 2, j}^{x}-Q_{i-1 / 2, j}^{x}}{\Delta x}+\frac{Q_{i, j+1 / 2}^{y}-Q_{i, j-1 / 2}^{y}}{\Delta y} \\
& =\frac{D_{i+1 / 2, j}^{x} \llbracket v \rrbracket_{i+1 / 2, j}^{2}+D_{i-1 / 2, j}^{x} \llbracket v \rrbracket_{i-1 / 2, j}^{2}}{2 \Delta x}+\frac{D_{i, j+1 / 2}^{y} \llbracket v \rrbracket_{i, j+1 / 2}^{2}+D_{i, j-1 / 2}^{y} \llbracket v \rrbracket_{i, j-1 / 2}^{2}}{2 \Delta y} .
\end{aligned}
$$

Under further assumptions on $\eta$ and $D$, this yields explicit bounds on "weak TV" terms of the form $\sum_{i, j} \llbracket v \rrbracket_{i+1 / 2, j}^{2} \Delta y$, which can be used to prove compactness and convergence of the numerical method; see e.g. [4]. We will apply this approach in Section 3.

Remark 2.3. The above observations can be used to design entropy stable schemes, by first finding numerical fluxes $\tilde{F}^{x}, \tilde{F}^{y}$ satisfying (2.9), and then adding diffusion in the form (2.8). We note that with this approach, we are only guaranteed that the discrete entropy inequality (2.4) (or (2.3) for entropy conservative schemes) is satisfied for one particular entropy pair $(\eta, q)$.

\subsection{The TECNO scheme}

The scheme (2.2) with fluxes $\tilde{F}^{x}, \tilde{F}^{y}$ satisfying (2.9) is entropy conservative, in the sense of Definition 2.1. It can be shown that two-point entropy conservative schemes are at most second-order accurate, in the sense of truncation error [19,20]. When adding diffusion in the form (2.8) with $D=O(1)$, the resulting scheme is at most first-order accurate. The TECNO schemes, introduced in [4,7], represent a systematic approach to designing higher-order accurate entropy stable schemes. Since the convergence proof in Section 3 only applies to the second-order TECNO schemes, we will only describe these methods here, and we refer to [4,7] for the general construction.

The TECNO scheme has two main ingredients: An entropy conservative flux $\tilde{F}^{x}, \tilde{F}^{y}$, and a sign preserving reconstruction method. Since our mesh is a Cartesian grid, we define the reconstruction procedure in a tensorial manner. For a partition $\left(\mathcal{C}_{i}\right)_{i \in \mathbb{Z}}$ of $\mathbb{R}$ we consider a $p$ th order reconstruction operator $\mathcal{R}$, mapping any grid function $\left(w_{i}\right)_{i \in \mathbb{Z}}$ to a piecewise $(p-1)$ th order polynomial $\mathcal{R} w(x)$. Multi-dimensional grid functions $\left(w_{i, j}\right)_{i, j \in \mathbb{Z}}$ are reconstructed dimension-by-dimension, defining in particular the edge values

$$
w_{i+1 / 2, j}^{ \pm}=\mathcal{R} w_{\cdot, j}\left(x_{i+1 / 2} \pm 0\right), \quad w_{i, j+1 / 2}^{ \pm}=\mathcal{R} w_{i, \cdot}\left(y_{j+1 / 2} \pm 0\right)
$$

(where we by "+0" and "-0" mean right and left limits, respectively). We define also the edge jumps

$$
\langle w\rangle_{i+1 / 2, j}=w_{i+1 / 2, j}^{+}-w_{i+1 / 2, j}^{-}, \quad\left\langle\langle w\rangle_{i, j+1 / 2}=w_{i, j+1 / 2}^{+}-w_{i, j+1 / 2}^{-} .\right.
$$

Fix now some entropy pair $(\tilde{\eta}, \tilde{q})$. The second-order TECNO scheme [4,7] is constructed from a flux $\tilde{F}^{x}, \tilde{F}^{y}$ which is entropy conservative with respect to $(\tilde{\eta}, \tilde{q})$, and applies a second-order reconstruction method to the 
entropy variables $\tilde{v}=\tilde{\eta} \circ u$. The resulting scheme (2.2) has numerical flux

$$
\begin{aligned}
& F_{i+1 / 2, j}^{x}=\tilde{F}_{i+1 / 2, j}-D_{i+1 / 2, j}^{x}\langle\tilde{v}\rangle_{i+1 / 2, j}, \\
& F_{i, j+1 / 2}^{y}=\tilde{F}_{i, j+1 / 2}-D_{i, j+1 / 2}^{y}\langle\tilde{v}\rangle_{i, j+1 / 2},
\end{aligned}
$$

where $D^{x}, D^{y} \geqslant 0$. As shown in [7], the above scheme is formally second-order accurate, and it satisfies the discrete entropy inequality (2.4) for the entropy pair $(\tilde{\eta}, \tilde{q})$, provided the reconstruction operator $\mathcal{R}$ satisfies the sign property

$$
\llbracket \tilde{v} \rrbracket_{i+1 / 2, j}\left\langle\langle\tilde{v}\rangle_{i+1 / 2, j} \geqslant 0, \quad \llbracket \tilde{v} \rrbracket_{i, j+1 / 2}\left\langle\langle\tilde{v}\rangle_{i, j+1 / 2} \geqslant 0 .\right.\right.
$$

This is indeed true for the ENO reconstruction method [11]:

Theorem 2.4 (The ENO sign property [8]). For some $p \in \mathbb{N}$, let $\mathcal{R}$ denote the $p$-th order ENO reconstruction

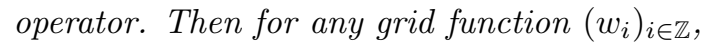

$$
\operatorname{sign}\left\langle\langle w\rangle_{i+1 / 2}=\operatorname{sign} \llbracket w \rrbracket_{i+1 / 2} .\right.
$$

Moreover, there exists a constant $C_{p}>0$ depending only on $p$ such that

$$
\frac{\langle w\rangle_{i+1 / 2}}{\llbracket w \rrbracket_{i+1 / 2}} \leqslant C_{p} .
$$

For the sake of simplicity we henceforth select the entropy for which TECNO is entropy stable as $\tilde{\eta}(u)=u^{2} / 2$. The corresponding entropy variable is then simply $\tilde{v}=u$, making the mapping between conserved and entropy variables somewhat easier. A summary of the TECNO scheme that we will analyze in this paper follows:

Definition 2.5. The second-order TECNO scheme for (2.1) is the numerical scheme (2.2) with the numerical flux

$$
\begin{aligned}
& F_{i+1 / 2, j}^{x}=\tilde{F}_{i+1 / 2, j}-D_{i+1 / 2, j}^{x}\langle u\rangle_{i+1 / 2, j}, \\
& F_{i, j+1 / 2}^{y}=\tilde{F}_{i, j+1 / 2}-D_{i, j+1 / 2}^{y}\langle u\rangle_{i, j+1 / 2},
\end{aligned}
$$

where

- $\tilde{F}$ is a consistent and locally Lipschitz continuous numerical flux which is entropy conservative with respect to the entropy $\tilde{\eta}(u)=u^{2} / 2$

- the diffusion coefficients D satisfy

$$
\underline{D} \leqslant D_{i+1 / 2, j}^{x}, D_{i, j+1 / 2}^{y} \leqslant \bar{D} \quad \text { for fixed } \underline{D}, \bar{D}>0
$$

- $\langle u\rangle_{i+1 / 2, j}$ and $\langle u\rangle_{i, j+1 / 2}$ denote the jumps in the second-order ENO reconstruction of the conserved variable $\left(u_{i, j}(t)\right)_{i, j \in \mathbb{Z}}$.

Before stating the next theorem, it should be mentioned once and for all that here and in the later sections, $C$ has been used as a generic constant which is independent of $\Delta x$ and $\Delta y$, but might vary in different calculations.

Theorem 2.6. The second-order TECNO scheme (cf. Definition 2.5) has the following properties:

(i) it is entropy stable with respect to the square entropy $\tilde{\eta}(u)=u^{2} / 2$

(ii) the flux $F$ is locally Lipschitz continuous

(iii) there is some $C>0$ independent of $\Delta x, \Delta y$ such that

$$
\int_{0}^{T} \sum_{i, j}\left(\left|\llbracket u \rrbracket_{i+1 / 2, j}\right|^{3} \Delta y+\left|\llbracket u \rrbracket_{i, j+1 / 2}\right|^{3} \Delta x\right) d t \leqslant C\|u\|_{L^{\infty}\left(\mathbb{R}^{2} \times[0, T]\right)},
$$




$$
\int_{0}^{T} \sum_{i, j}\left(\llbracket u \rrbracket_{i+1 / 2, j}\langle u\rangle_{i+1 / 2, j} \Delta y+\llbracket u \rrbracket_{i, j+1 / 2}\langle u\rangle_{i, j+1 / 2} \Delta x\right) d t \leqslant C .
$$

where $u=\left(u_{i, j}(t)\right)_{i, j}$ is the solution computed by the scheme.

Proof. The entropy stability follows from the calculations in Section 2.2 and the sign property (2.12). Local Lipschitz continuity of $F$ follows from the Lipschitz continuity of $\tilde{F}$ and the upper bound (2.13).

For the "weak TV bounds" (2.15), summing (2.6) over $i, j \in \mathbb{Z}$, integrating over $t \in[0, T]$ and using the specific form of $F$ in (2.14) yields

$$
\begin{array}{r}
\frac{1}{2} \sum_{i, j}\left(u_{i, j}(T)\right)^{2} \Delta x \Delta y-\frac{1}{2} \sum_{i, j}\left(u_{i, j}(0)\right)^{2} \Delta x \Delta y=-\mathcal{E}, \\
\mathcal{E}:=\int_{0}^{T} \sum_{i, j}\left(D_{i+1 / 2, j}^{x} \llbracket u \rrbracket_{i+1 / 2, j}\langle u\rangle_{i+1 / 2, j} \Delta y+D_{i, j+1 / 2}^{y} \llbracket u \rrbracket_{i, j+1 / 2}\langle u\rangle_{i, j+1 / 2} \Delta x\right) d t .
\end{array}
$$

Since $D \geqslant 0$ and the reconstruction satisfies the sign property (2.12), we have $\mathcal{E} \geqslant 0$. From the above we also see that $\mathcal{E} \leqslant \frac{1}{2} \sum_{i, j}\left(u_{i, j}(0)\right)^{2} \Delta x \Delta y \leqslant \frac{1}{2}\left\|u_{0}\right\|_{L^{2}\left(\mathbb{R}^{2}\right)}^{2}$, so we see that the left-hand side of (2.15b) can be upper-bounded by $\underline{D} \mathcal{E} \leqslant \frac{1}{2} \underline{D}\left\|u_{0}\right\|_{L^{2}\left(\mathbb{R}^{2}\right)}^{2}<\infty$.

For the remaining property (2.15a) we use the following fact, proved in [5, Section 4.4]: For every grid function $\left(w_{i}\right)_{i \in \mathbb{Z}}$ with compact support,

$$
\sum_{i \in \mathbb{Z}}\left|\llbracket w \rrbracket_{i+1 / 2}\right|^{3} \leqslant 2\|w\|_{l \infty} \sum_{i \in \mathbb{Z}}\left\langle\langle w\rangle_{i+1 / 2} \llbracket w \rrbracket_{i+1 / 2}\right.
$$

where $\langle w\rangle_{i+1 / 2}=w_{i+1 / 2}^{+}-w_{i+1 / 2}^{-}$denotes the jump in the second-order ENO reconstruction of $w$. Thus, the left-hand side of $(2.15 \mathrm{a})$ can be bounded by $\|u\|_{L^{\infty}}$ times the left-hand side of $(2.15 \mathrm{~b})$.

Remark 2.7. For even higher-order TECNO schemes, the results in Theorem 2.6 are still valid, with the exception of (2.15a): The crucial estimate (2.16) has been conjectured but remains unproven; cf. [5, Section 4.4] or [4, Section 5.5].

\section{Convergence of the scheme}

Given a numerical solution $\left(u_{i, j}(t)\right)_{i, j \in \mathbb{Z}, t \in \mathbb{R}_{+}}$computed with the second-order TECNO scheme (cf. Definition $2.5)$ we define the piecewise constant function

$$
u^{\Delta}(x, y, t):=u_{i, j}(t) \quad \text { for }(x, y) \in \mathcal{C}_{i, j}
$$

where $\boldsymbol{\Delta}=(\Delta x, \Delta y)$. The goal of this section will be to show the following theorem:

Theorem 3.1. Assume that the solution $u^{\Delta}$ computed by the TECNO scheme (cf. Definition 2.5) is uniformly $L^{\infty}$ bounded,

$$
\left\|u^{\boldsymbol{\Delta}}\right\|_{L^{\infty}\left(\mathbb{R}^{2} \times[0, T]\right)} \leqslant M \quad \text { for every } \boldsymbol{\Delta}=(\Delta x, \Delta y)>0
$$

for some $M>0$. Then there is some subsequence $\boldsymbol{\Delta}^{\prime} \rightarrow 0$ such that $u^{\boldsymbol{\Delta}^{\prime}} \rightarrow$ u pointwise a.e. and in $L^{p}\left(\mathbb{R}^{2} \times[0, T]\right)$ for every $p \in[1, \infty)$. The function $u$ is a weak solution of (2.1) which satisfies the entropy condition (1.5) for the entropy $\eta(u)=u^{2}$.

We will use the method of compensated compactness, and we give the main results required here in Section 3.1. The convergence proof is given in Section 3.2, but we summarize it here: 
Proof of Theorem 3.1. The compactness result, Corollary 3.4 requires the entropy residuals $\left\{\partial_{t} \eta\left(u^{\boldsymbol{\Delta}}\right)+\nabla\right.$. $\left.q\left(u^{\boldsymbol{\Delta}}\right)\right\}_{\boldsymbol{\Delta}>0}$ to lie in a compact subset of $H_{\text {loc }}^{-1}$. Lemma 3.5 bounds their discrete equivalents $\partial_{t} \eta\left(u^{\boldsymbol{\Delta}}\right)+\nabla \cdot Q\left(u^{\boldsymbol{\Delta}}\right)$ by terms which, by Theorem 2.6(iii) and (3.1), are bounded in the sense of measures. Lemma 3.6 shows that the remainder $\nabla \cdot(q-Q)$ is small in $H_{\text {loc }}^{-1}$. We then conclude (using Murat's Lemma) that the entropy residuals are precompact, and hence there is some strongly convergent subsequence.

Lemma 3.7 is a standard "Lax-Wendroff" proof, showing that the limit is a weak solution, and Lemma 3.8 shows consistency with a single entropy condition.

\subsection{Compensated compactness}

We briefly summarize the technical compactness lemmas here, and refer to $[1,22]$ for more details.

Lemma 3.2 (Murat's Lemma). Let $\Omega \subset \mathbb{R}^{d}, d \geqslant 2$ be an open, bounded set. Let $(\mu)_{n \in \mathbb{N}}$ be a bounded sequence in $W^{-1, p}(\Omega)$ for some $2<p \leqslant \infty$. Suppose also that $\forall n \in \mathbb{N}$

$$
\mu_{n}=\xi_{n}+\pi_{n},
$$

where $\xi_{n}$ lies in a compact set of $H_{\text {loc }}^{-1}(\Omega)$ and $\pi_{n}$ lies in a bounded set of $\mathcal{M}_{\text {loc }}(\Omega)$. Then $\left(\mu_{n}\right)_{n \in \mathbb{N}}$ lies in a compact subset of $H_{l o c}^{-1}(\Omega)$.

Theorem 3.3 (Panov, Theorem 5 in [18]). Let $\left(u_{\varepsilon}\right)_{\varepsilon>0}$ be a bounded sequence in $L^{\infty}\left(\mathbb{R}^{d} \times \mathbb{R}_{+}\right)$such that for every $k \in \mathbb{R}$, the set

$$
\left\{\partial_{t} \eta_{k}\left(u_{\varepsilon}\right)+\nabla \cdot q_{k}\left(u_{\varepsilon}\right)\right\}_{\varepsilon>0}
$$

is precompact in $H_{\text {loc }}^{-1}\left(\mathbb{R}^{d} \times \mathbb{R}_{+}\right)$. (Here, $\left(\eta_{k}, q_{k}\right)$ denote the Kruzkov entropy pairs (1.3).) Then there is a subsequence $\varepsilon_{n} \rightarrow 0$ as $n \rightarrow \infty$ and a function $u \in L^{\infty}\left(\mathbb{R}^{d} \times \mathbb{R}_{+}\right)$such that

$$
u_{\varepsilon_{n}} \rightarrow u \quad \text { a.e. and in } L_{\text {loc }}^{p}\left(\mathbb{R}^{d} \times \mathbb{R}_{+}\right) \text {for every } 1 \leqslant p<\infty .
$$

The following corollary shows that it is enough to consider smooth entropies in the above result.

Corollary 3.4. Let $\left(u_{\varepsilon}\right)_{\varepsilon>0}$ be a bounded sequence in $L^{\infty}\left(\mathbb{R}^{d} \times \mathbb{R}_{+}\right)$such that for every entropy pair $(\eta, q)$ with $\eta \in C_{b}^{2}(\mathbb{R})$, the set

$$
\left\{\partial_{t} \eta\left(u_{\varepsilon}\right)+\nabla \cdot q\left(u_{\varepsilon}\right)\right\}_{\varepsilon>0}
$$

is precompact in $H_{\text {loc }}^{-1}\left(\mathbb{R}^{d} \times \mathbb{R}_{+}\right)$. Then there is a subsequence $\varepsilon_{n} \rightarrow 0$ as $n \rightarrow \infty$ and a function $u \in L^{\infty}\left(\mathbb{R}^{d} \times \mathbb{R}_{+}\right)$ such that

$$
u_{\varepsilon_{n}} \rightarrow u \text { a.e. and in } L_{\text {loc }}^{p}\left(\mathbb{R}^{d} \times \mathbb{R}_{+}\right) \text {for every } 1 \leqslant p<\infty .
$$

Proof. Since this result is rather standard we omit a full proof, but the idea is to approximate $\eta_{k}$ by $C^{2}$ convex entropies which converge uniformly to $\eta_{k}$.

\subsection{Convergence of TECNO}

The TECNO scheme (Definition 2.5) is guaranteed to dissipate the square entropy $\tilde{\eta}(u)=u^{2} / 2$, but for an arbitrary entropy $\eta$ the corresponding discrete entropy residual (2.6) might have either sign. We can nonetheless show that the entropy residual is not too large, in the following sense:

Lemma 3.5. Assume that the solution computed by the TECNO scheme is $L^{\infty}$ bounded, (3.1). Then for any entropy pair $(\eta, q)$ with $\eta \in C^{2}$, the total discrete entropy production is upper-bounded by

$$
\begin{aligned}
& \mid \partial_{t} \eta\left(u^{\Delta}\right)+\nabla \cdot Q \mid\left(\mathbb{R}^{2} \times[0, T]\right) \leqslant C \int_{0}^{T} \sum_{i, j}\left(\left|\llbracket u \rrbracket_{i+1 / 2, j}\right|^{3} \Delta y+\left|\llbracket u \rrbracket_{i, j+1 / 2}\right|^{3} \Delta x\right) d t \\
&+C \int_{0}^{T} \sum_{i, j}\left(\llbracket u \rrbracket_{i+1 / 2, j}\langle u\rangle_{i+1 / 2, j} \Delta y+\llbracket u \rrbracket_{i, j+1 / 2}\left\langle\langle u\rangle_{i, j+1 / 2} \Delta x\right) d t\right.
\end{aligned}
$$


where $\nabla \cdot Q$ denotes the measure whose integral of any $\varphi \in C_{c}^{0}\left(\mathbb{R}^{2} \times \mathbb{R}_{+}\right)$is

$$
\begin{aligned}
\langle\nabla \cdot Q, \varphi\rangle & =\sum_{i, j \in \mathbb{Z}} \int_{\mathbb{R}_{+}} \bar{\varphi}_{i+1 / 2, j} \frac{Q_{i+1 / 2, j}^{x}-Q_{i-1 / 2, j}^{x}}{\Delta x}+\bar{\varphi}_{i, j+1 / 2} \frac{Q_{i, j+1 / 2}^{y}-Q_{i, j-1 / 2}^{y}}{\Delta y} d t \Delta x \Delta y, \\
\bar{\varphi}_{i+1 / 2, j} & :=\frac{1}{\Delta y} \int_{y_{j-1 / 2}}^{y_{j+1 / 2}} \varphi\left(x_{i+1 / 2}, y, t\right) d y, \quad \bar{\varphi}_{i, j+1 / 2}:=\frac{1}{\Delta x} \int_{x_{i-1 / 2}}^{x_{i+1 / 2}} \varphi\left(x, y_{j+1 / 2}, t\right) d x
\end{aligned}
$$

and where $Q^{x}, Q^{y}$ are given by (2.7).

Proof. The technique of the proof is to compare the entropy residual of an arbitrary entropy $\eta$ to the entropy residual of the square entropy. Let $(\eta, q)$ be any entropy pair with corresponding entropy residual $\psi=\left(\psi^{x}, \psi^{y}\right)$, and let $\tilde{\psi}$ be the entropy potential with respect to the square entropy $\tilde{\eta}=u^{2} / 2, \tilde{\psi}(u)=u f(u)-\tilde{q}(u)$. We split the entropy residual $r$ in (2.7) as $r=r^{1}+r^{2}$, where

$$
\left.r_{i+1 / 2, j}^{1}=\llbracket v \rrbracket_{i+1 / 2, j} \tilde{F}_{i+1 / 2, j}^{x}-\llbracket \psi^{x} \rrbracket_{i+1 / 2, j}, \quad r_{i+1 / 2, j}^{2}=-\llbracket v \rrbracket_{i+1 / 2, j} D_{i+1 / 2}^{x} \| u\right\rangle_{i+1 / 2, j}
$$

and similarly for $r_{i, j+1 / 2}^{y}$. If $\llbracket u \rrbracket_{i+1 / 2, j}=0$ then $r_{i+1 / 2, j}=0$, so assume $\llbracket u \rrbracket_{i+1 / 2, j} \neq 0$. The first part of the entropy residual can be estimated as

$$
\begin{aligned}
\left|r_{i+1 / 2, j}^{1}\right| & \leqslant\left|\llbracket v \rrbracket_{i+1 / 2, j} \tilde{F}_{i+1 / 2, j}^{x}-\llbracket \psi^{x} \rrbracket_{i+1 / 2, j}\right| \\
& =\left|\llbracket v \rrbracket_{i+1 / 2, j}\left(\frac{\llbracket \tilde{\psi}^{x} \rrbracket_{i+1 / 2, j}}{\llbracket u \rrbracket_{i+1 / 2, j}}-\frac{\llbracket \psi^{x} \rrbracket_{i+1 / 2, j}}{\llbracket v \rrbracket_{i+1 / 2, j}}\right)\right| \\
& =\left|\llbracket v \rrbracket_{i+1 / 2, j}\right|\left|\frac{1}{\llbracket u \rrbracket_{i+1 / 2, j}} \int_{u_{i, j}}^{u_{i+1, j}}\left(\tilde{\psi}^{x}\right)^{\prime}(v) d v-\frac{1}{\llbracket v \rrbracket_{i+1 / 2, j}} \int_{v_{i, j}}^{v_{i+1, j}}\left(\psi^{x}\right)^{\prime}(v) d v\right| \\
& =\left|\llbracket v \rrbracket_{i+1 / 2, j}\right|\left|\frac{1}{\llbracket u \rrbracket_{i+1 / 2, j}} \int_{u_{i, j}}^{u_{i+1, j}} f^{x}(u) d u-\frac{1}{\llbracket v \rrbracket_{i+1 / 2, j}} \int_{v_{i, j}}^{v_{i+1, j}} f^{x}(u(v)) d v\right|
\end{aligned}
$$

(by the mean value theorem)

$$
\begin{gathered}
=\left|\llbracket v \rrbracket_{i+1 / 2, j}\right| \mid \frac{f^{x}\left(u_{i, j}\right)+f^{x}\left(u_{i+1, j}\right)}{2}-\frac{\llbracket u \rrbracket_{i+1 / 2, j}^{2}}{12}\left(\tilde{\psi}^{x}\right)^{\prime \prime \prime}\left(\tilde{\xi}_{i+1 / 2, j}\right) \\
-\frac{f^{x}\left(u_{i, j}\right)+f^{x}\left(u_{i+1, j}\right)}{2}+\frac{\llbracket v \rrbracket_{i+1 / 2, j}^{2}}{12}\left(\psi^{x}\right)^{\prime \prime \prime}\left(\xi_{i+1 / 2, j}\right) \mid
\end{gathered}
$$

(by the $L^{\infty}$ bound on $u$ )

$$
\leqslant C\left|\llbracket u \rrbracket_{i+1 / 2, j}\right|^{3},
$$

and similarly in the $y$-direction,

$$
\left|r_{i, j+1 / 2}^{1}\right| \leqslant C\left|\llbracket u \rrbracket_{i, j+1 / 2}\right|^{3} .
$$

The second part of the entropy residual can be bounded by

$$
\left|r_{i+1 / 2, j}^{2}\right| \leqslant\left\|\eta^{\prime \prime}\right\|_{L^{\infty}([-M, M])} \bar{D} \llbracket u \rrbracket_{i+1 / 2}\langle u u\rangle_{i+1 / 2} .
$$

The conclusion now follows. 
We can now show precompactness of the sequence of approximations:

Lemma 3.6. Let $\Omega \subset \mathbb{R}^{2} \times[0, T]$ be a bounded subset and assume that the solution computed by the TECNO scheme is $L^{\infty}$ bounded, (3.1). Then there is a subsequence $\boldsymbol{\Delta}^{\prime} \rightarrow 0$ such that $u^{\boldsymbol{\Delta}^{\prime}} \rightarrow u$ pointwise a.e. and in $L_{\text {loc }}^{p}\left(\mathbb{R}^{2} \times \mathbb{R}_{+}\right)$for $1 \leqslant p<\infty$, for some $u \in L^{1} \cap L^{\infty}\left(\mathbb{R}^{2} \times \mathbb{R}_{+}\right)$.

Proof. Let $(\eta, q)$ be an arbitrary $C^{2}$ entropy pair. By Corollary 3.4 it is sufficient to show that the sequence $\mathcal{E}_{\Delta x, \Delta y}:=\partial_{t} \eta\left(u^{\boldsymbol{\Delta}}\right)+\nabla \cdot q\left(u^{\boldsymbol{\Delta}}\right)$ is precompact in $H_{\text {loc }}^{-1}$, and to this end we will employ Murat's lemma. Firstly note that $\mathcal{E}_{\Delta x, \Delta y}$ is bounded in $W^{-1, \infty}\left(\mathbb{R}^{2} \times \mathbb{R}_{+}\right)$, by the $L^{\infty}$ bound on $u^{\boldsymbol{\Delta}}$. Decompose

$$
\partial_{t} \eta(u)+\nabla \cdot q(u)=\underbrace{\partial_{t} \eta(u)+\nabla \cdot Q}_{=: \mathcal{E}^{1}}+\underbrace{\nabla \cdot q(u)-\nabla \cdot Q}_{=: \mathcal{E}^{2}}
$$

where $Q$ is given in (2.7). Note that, due to the $L^{\infty}$ bound on $u^{\Delta}$ and Theorem 2.6, also $Q$ is locally Lipschitz continuous. By Lemma 3.5 and Theorem 2.6(iii), the discrete entropy production $\mathcal{E}^{1}$ is bounded in the space of measures $\mathcal{M}\left(\mathbb{R}^{d} \times \mathbb{R}_{+}\right)$.

Now to show that $\mathcal{E}^{2}$ is precompact in $H_{\text {loc }}^{-1}\left(\mathbb{R}^{2} \times[0, T]\right)$, let $\Omega \subset \mathbb{R}^{2} \times[0, T]$ be open and bounded and let $\varphi \in H_{0}^{1}(\Omega)$. Extending $\varphi$ by zero outside $\Omega$, we get

$$
\mathcal{E}^{2}(\varphi)=\int_{\Omega} \varphi d(\nabla \cdot q(u)-\nabla \cdot Q) d(x, y, t)
$$

$$
\begin{aligned}
= & \int_{0}^{T} \sum_{i, j} \bar{\varphi}_{i+1 / 2, j}\left(q^{x}\left(u_{i+1, j}\right)-q^{x}\left(u_{i, j}\right)\right) \Delta y+\bar{\varphi}_{i, j+1 / 2}\left(q^{y}\left(u_{i, j+1}\right)-q^{y}\left(u_{i, j}\right)\right) \Delta x d t \\
& -\int_{0}^{T} \sum_{i, j \in \mathbb{Z}} \bar{\varphi}_{i+1 / 2, j} \frac{Q_{i+1 / 2, j}^{x}-Q_{i-1 / 2, j}^{x}}{\Delta x}+\bar{\varphi}_{i, j+1 / 2} \frac{Q_{i, j+1 / 2}^{y}-Q_{i, j-1 / 2}^{y}}{\Delta y} d t \Delta x \Delta y
\end{aligned}
$$

(summation by parts)

$$
\begin{aligned}
= & \int_{0}^{T} \sum_{i, j} \frac{\bar{\varphi}_{i+1 / 2, j}-\bar{\varphi}_{i-1 / 2, j}}{\Delta x}\left(Q_{i-1 / 2, j}^{x}-q^{x}\left(u_{i, j}\right)\right) d t \Delta x \Delta y \\
& +\int_{0}^{T} \sum_{i, j} \frac{\bar{\varphi}_{i, j+1 / 2}-\bar{\varphi}_{i, j-1 / 2}}{\Delta y}\left(Q_{i, j-1 / 2}^{y}-q^{y}\left(u_{i, j}\right)\right) d t \Delta x \Delta y
\end{aligned}
$$

(letting $\left.\mathcal{I}=\left\{(i, j): \Omega \cap \mathcal{C}_{i, j} \neq \emptyset\right\}\right)$

$$
\begin{aligned}
\leqslant & \left\|\partial_{x} \varphi\right\|_{L^{2}(\Omega)}\left(\int_{0}^{T} \sum_{(i, j) \in \mathcal{I}}\left|Q_{i-1 / 2, j}^{x}-q^{x}\left(u_{i, j}\right)\right|^{2} \Delta x \Delta y d t\right)^{\frac{1}{2}} \\
& +\left\|\partial_{y} \varphi\right\|_{L^{2}(\Omega)}\left(\int_{0}^{T} \sum_{(i, j) \in \mathcal{I}}\left|Q_{i, j-1 / 2}^{y}-q^{y}\left(u_{i, j}\right)\right|^{2} \Delta x \Delta y d t\right)^{\frac{1}{2}}
\end{aligned}
$$


(by Lipschitz continuity of $Q$ )

$$
\begin{aligned}
& \leqslant C\|\varphi\|_{H^{1}(\Omega)}\left[\left(\int_{0}^{T} \sum_{(i, j) \in \mathcal{I}}\left|\llbracket u \rrbracket_{i+1 / 2, j}\right|^{2} \Delta x \Delta y d t\right)^{\frac{1}{2}}+\left(\int_{0}^{T} \sum_{(i, j) \in \mathcal{I}}\left|\llbracket u \rrbracket_{i, j+1 / 2}\right|^{2} \Delta x \Delta y d t\right)^{\frac{1}{2}}\right] \\
& \leqslant C\|\varphi\|_{H^{1}(\Omega)}|\Omega|^{\frac{3}{2}}\left[\left(\int_{0}^{T} \sum_{i, j}\left|\llbracket u \rrbracket_{i+1 / 2, j}\right|^{3} \Delta x \Delta y d t\right)^{\frac{1}{3}}+\left(\int_{0}^{T} \sum_{i, j}\left|\llbracket u \rrbracket_{i, j+1 / 2}\right|^{3} \Delta x \Delta y d t\right)^{\frac{1}{3}}\right] \\
& \rightarrow 0
\end{aligned}
$$

where the last step follows from (2.15). Thus by invoking Murat's Lemma 3.2 we can conclude that the sequence $\left(\mathcal{E}_{\Delta x, \Delta y}\right)_{\Delta x, \Delta y>0}$ is precompact in $H_{\text {loc }}^{-1}\left(\mathbb{R}^{2} \times[0, T]\right)$. Applying Corollary 3.4 then yields the desired result.

Now we need to show that this limit function $u$ is indeed a weak solution of (2.1). To do so we state and prove the following "Lax-Wendroff result".

Lemma 3.7. Under the same assumptions as in Lemma 3.6, the limit $u$ is a weak solution of (2.1).

Proof. Let $\varphi \in C_{c}^{1}\left(\mathbb{R}^{2} \times(0, T)\right)$ be a test function and select a compact set $K_{x} \times K_{y} \subset \mathbb{R}^{2}$ such that $\operatorname{supp} \varphi \subset$ $K_{x} \times K_{y} \times[0, T]$. Furthermore, denote

$$
\varphi_{i, j}(t)=\varphi\left(x_{i}, y_{j}, t\right), \quad \varphi^{\Delta}(x, y, t)=\sum_{i, j} \varphi_{i, j}(t) \chi_{\mathcal{C}_{i, j}}(x, y)
$$

Multiplying the numerical scheme (2.2) by $\varphi_{i, j}(t)$ and integrating/summing in time/space, we get

$$
\begin{aligned}
0= & \int_{0}^{T} \Delta x \Delta y \sum_{i, j}\left(\varphi_{i, j} \frac{d}{d t} u_{i, j}^{\Delta}+\varphi_{i, j} \frac{\tilde{F}_{i+1 / 2, j}^{x}-\tilde{F}_{i-1 / 2, j}^{x}}{\Delta x}+\varphi_{i, j} \frac{\tilde{F}_{i, j+1 / 2}^{y}-\tilde{F}_{i, j-1 / 2}^{y}}{\Delta y}\right. \\
& -\varphi_{i, j} \frac{D_{i+1 / 2, j}^{x}\left\langle\langle u\rangle_{i+1 / 2, j}-D_{i-1 / 2, j}^{x}\langle u\rangle_{i-1 / 2, j}\right.}{\Delta x} \\
& \left.-\varphi_{i, j} \frac{D_{i, j+1 / 2}^{y}\langle u\rangle_{i, j+1 / 2}-D_{i, j-1 / 2}^{y}\langle u\rangle_{i, j-1 / 2}}{\Delta y}\right) d t .
\end{aligned}
$$

After performing integration and summation by parts for temporal and spatial variables respectively we get

$$
A^{1}+A^{2}+A^{3}+B^{1}+B^{2}=0,
$$

where we can write

$$
\begin{aligned}
A^{1} & :=-\int_{0}^{T} \Delta x \Delta y \sum_{i, j} u_{i, j} \frac{d}{d t} \varphi_{i, j} d t \\
A^{2} & :=-\int_{0}^{T} \Delta x \Delta y \sum_{i, j} \tilde{F}_{i+1 / 2, j}^{x} \frac{\varphi_{i+1, j}-\varphi_{i, j}}{\Delta x} d t, \\
A^{3} & :=-\int_{0}^{T} \Delta x \Delta y \sum_{i, j} \tilde{F}_{i, j+1 / 2}^{y} \frac{\varphi_{i, j+1}-\varphi_{i, j}}{\Delta y} d t, \\
B^{1} & :=\int_{0}^{T} \Delta x \Delta y \sum_{i, j} D_{i+1 / 2, j}^{x}\langle u\rangle_{i+1 / 2, j} \frac{\varphi_{i+1, j}-\varphi_{i, j}}{\Delta x} d t,
\end{aligned}
$$




$$
B^{2}:=\int_{0}^{T} \Delta x \Delta y \sum_{i, j} D_{i, j+1 / 2}^{y}\left\langle\langle u\rangle_{i, j+1 / 2} \frac{\varphi_{i, j+1}-\varphi_{i, j}}{\Delta y} d t .\right.
$$

We can write $A^{1}=-\int_{0}^{T} \int_{\mathbb{R}} \int_{\mathbb{R}} u^{\boldsymbol{\Delta}} \partial_{t} \varphi^{\boldsymbol{\Delta}} d x d y d t$, and thanks to the convergence of $u^{\boldsymbol{\Delta}}$ to $u$ from Lemma 3.6 and convergence of $\varphi^{\Delta}$ to $\varphi$ a.e., we have $\lim _{\Delta x, \Delta y \rightarrow 0} A^{1}=-\int_{0}^{T} \int_{\mathbb{R}^{2}} u \partial_{t} \varphi d x d y d t$.

For the second term $A^{2}$, we denote for the sake of simplicity $\Delta_{x} \psi(x, y, t)=\frac{\psi(x+\Delta x, y, t)-\psi(x, y, t)}{\Delta x}$, for any function $\psi$. Since $\tilde{F}^{x}$ is a two-point flux, we can write

$$
\begin{aligned}
A^{2} & =-\int_{0}^{T} \int_{\mathbb{R}^{2}} \tilde{F}^{x}\left(u^{\Delta}(x, y, t), u^{\Delta}(x+\Delta x, y, t)\right) \Delta_{x} \varphi^{\Delta}(x, y, t) d(x, y) d t \\
& =A^{2,1}+A^{2,2}
\end{aligned}
$$

where

$$
\begin{aligned}
& A^{2,1}:=-\int_{0}^{T} \int_{\mathbb{R}^{2}} f^{x}\left(u^{\boldsymbol{\Delta}}(x, y, t)\right) \Delta_{x} \varphi^{\boldsymbol{\Delta}}(x, y, t) d(x, y) d t \\
& A^{2,2}:=\int_{0}^{T} \int_{\mathbb{R}^{2}}\left(f^{x}\left(u^{\boldsymbol{\Delta}}(x, y, t)\right)-\tilde{F}^{x}\left(u^{\boldsymbol{\Delta}}(x, y, t), u^{\boldsymbol{\Delta}}(x+\Delta x, y, t)\right)\right) \Delta_{x} \varphi^{\boldsymbol{\Delta}}(x, y, t) d(x, y) d t .
\end{aligned}
$$

Thanks to the convergence of $u^{\boldsymbol{\Delta}}$ from Lemma 3.6 and the convergence of $\Delta_{x} \varphi^{\boldsymbol{\Delta}}$ to $\partial_{x} \varphi$ we have

$$
A^{2,1} \rightarrow-\int_{0}^{T} \int_{\mathbb{R}^{2}} f^{x}(u) \partial_{x} \varphi d(x, y) d t \quad \text { as } \boldsymbol{\Delta} \rightarrow 0
$$

For the term $A^{2,2}$ we apply the Hölder inequality and Lemma 3.6 to get

$$
\left|A^{2,2}\right| \leqslant \int_{0}^{T} \int_{\mathbb{R}^{2}}\left|\tilde{f}^{x}\left(u^{\boldsymbol{\Delta}}(x, y, t)\right)-\tilde{F}^{x}\left(u^{\boldsymbol{\Delta}}(x, y, t), u^{\boldsymbol{\Delta}}(x+\Delta x, y, t)\right)\right|\left|\Delta_{x} \varphi^{\boldsymbol{\Delta}}(x, y, t)\right| d(x, y) d t
$$

(using Lipschitz continuity of $\tilde{F}^{x}$ )

$$
\begin{aligned}
& \leqslant C \int_{0}^{T} \int_{\mathbb{R}^{2}}\left|u^{\Delta}(x+\Delta x, y, t)-u^{\Delta}(x, y, t)\right|\left|\Delta_{x} \varphi^{\Delta}(x, y, t)\right| d(x, y) d t \\
& \leqslant C\left(\int_{0}^{T} \int_{\mathbb{R}^{2}}\left|\Delta_{x} \varphi^{\Delta}\right|^{\frac{3}{2}} d(x, y) d t\right)^{\frac{2}{3}}\left(\int_{0}^{T} \sum_{i, j}\left|\llbracket u \rrbracket_{i+1 / 2, j}\right|^{3} \Delta x \Delta y d t\right)^{\frac{1}{3}} \\
& \leqslant C\left\|\partial_{x} \varphi\right\|_{L^{\frac{3}{2}}\left(\mathbb{R}^{2} \times[0, T)\right)}\left(\int_{0}^{T} \sum_{i, j}\left|\llbracket u \rrbracket_{i+1 / 2, j}\right|^{3} \Delta x \Delta y d t\right)^{\frac{1}{3}} \\
& \rightarrow 0
\end{aligned}
$$

as $\Delta x, \Delta y \rightarrow 0$ by (2.15a). Analogously, $A^{3} \rightarrow-\int_{0}^{T} \int_{\mathbb{R}^{2}} f^{y}(u) \partial_{y} \varphi d x d y d t$ as $\Delta x, \Delta y \rightarrow 0$. We conclude that

$$
A \rightarrow-\int_{0}^{T} \int_{\mathbb{R}^{2}}\left[u \partial_{t} \varphi+f(u) \cdot \nabla \varphi\right] d(x, y) d t \quad \text { as } \Delta x, \Delta y \rightarrow 0 .
$$


It remains to show that $B^{1}, B^{2}$ in (3.9) vanish as $\Delta x, \Delta y \rightarrow 0$. Indeed,

$$
\left|B^{1}\right| \leqslant \bar{D} \int_{0}^{T} \Delta x \Delta y \sum_{i, j}\left|\langle u\rangle_{i+1 / 2, j}\right|\left|\frac{\varphi_{i+1, j}-\varphi_{i, j}}{\Delta x}\right| d t
$$

(by $(2.13))$

$$
\begin{aligned}
& \leqslant C \bar{D}\left\|\eta^{\prime \prime}\right\|_{L^{\infty}(\mathbb{R})} \int_{0}^{T} \Delta x \Delta y \sum_{i, j}\left|\llbracket u \rrbracket_{i+1 / 2, j}\right|\left|\frac{\varphi_{i+1, j}-\varphi_{i, j}}{\Delta x}\right| d t \\
& \leqslant C \bar{D}\left\|\eta^{\prime \prime}\right\|_{L^{\infty}(\mathbb{R})}\left\|\partial_{x} \varphi\right\|_{L^{3 / 2}\left(\mathbb{R}^{2} \times[0, T]\right)}\left(\int_{0}^{T} \Delta x \Delta y \sum_{i, j}\left|\llbracket u \rrbracket_{i+1 / 2, j}\right|^{3} d t\right)^{1 / 3} \\
& \rightarrow 0
\end{aligned}
$$

as $\Delta x, \Delta y \rightarrow 0$, and likewise for $B^{2}$. This completes the proof.

Although we are not able to show that the TECNO scheme converges to the entropy solution, we will show that the weak solution $u$ satisfies at least one of the entropy conditions.

Lemma 3.8. With the same assumptions as in Lemma 3.6, the limit u satisfies

$$
\partial_{t} \tilde{\eta}(u)+\nabla \cdot \tilde{q}(u) \leqslant 0 .
$$

Proof. As in (2.6) in Lemma 3.5 we can write

$$
\frac{d}{d t} \tilde{\eta}\left(u_{i, j}\right)+\frac{\tilde{Q}_{i+1 / 2, j}^{x}-\tilde{Q}_{i-1 / 2, j}^{x}}{\Delta x}+\frac{\tilde{Q}_{i, j+1 / 2}^{y}-\tilde{Q}_{i, j-1 / 2}^{y}}{\Delta y}=\frac{r_{i+1 / 2, j}^{x}+r_{i-1 / 2, j}^{x}}{2 \Delta x}+\frac{r_{i, j+1 / 2}^{y}+r_{i, j-1 / 2}^{y}}{2 \Delta y}
$$

where, in this particular case, the entropy residuals $r$ on the right-hand side are all nonpositive (see e.g. [4,7,19]). Multiplying the above by a nonnegative test function $\varphi \in C_{c}^{1}\left(\mathbb{R}^{2} \times(0, T)\right)$ and proceeding in the same manner as in Lemma 3.7 we obtain (3.12) in the sense of distribution.

\section{Conclusions And outlook}

We prove convergence of the second-order, semi-discrete TECNO scheme in two space dimensions to a weak solution of the hyperbolic conservation law (1.1); this can easily be generalized to any number of space dimensions. The proof of this result relies on estimating the entropy residual appropriately using the (weak) TV bound obtained from entropy stability with respect to one entropy. Invoking this estimate, along with an assumption of $L^{\infty}$ boundedness, precompactness of the sequence of approximate solutions is shown using a corollary derived from a compensated compactness result due to Panov. Finally, to show that the limit function obtained due to the precompactness property is indeed a weak solution of (1.1), a "Lax-Wendroff" type argument is used.

Convergence proofs of even higher-order (i.e. more than second order) TECNO scheme in multiple space dimensions, to a weak solution of the equation (1.1) are still unanswered. In our opinion, this is largely due to the unavailability of weak TV estimates of the type (2.15a), as well as an appropriate version of Lemma 3.5. This should be an object of interest for future research. Last, but not least, one key estimate to prove (2.15b), and consequently (2.15a), is (2.16). For even higher-order ENO reconstruction, the estimate (2.16) (the "ENO-conjecture") is still not established and remains an open problem. 


\section{AcKnowledgement}

The work of this paper was initiated when N.C. and U.S.F. visited Seminar for Applied Mathematics (SAM), ETH Zürich. The authors would like to thank SAM for the warm hospitality and facilities, and Prof. Dr. Siddhartha Mishra for discussing the problem. N.C.'s work was funded by European Union's Horizon 2020 research and innovation programme under the Marie Skłodowska-Curie grant agreement No 642768.

\section{REFERENCES}

[1] G.Q. Chen and Y.G. Lu, The study on application way of the compensated compactness theory, Chinese Sci. Bull. 34 (1989), no. $1,15-19$.

[2] Tianheng Chen and Chi-Wang Shu, Entropy stable high order discontinuous Galerkin methods with suitable quadrature rules for hyperbolic conservation laws, J. Comput. Phys. 345 (2017), 427-461.

[3] C.M. Dafermos, Hyperbolic conservation laws in continuum physics, fourth ed., Grundlehren der Mathematischen Wissenschaften [Fundamental Principles of Mathematical Sciences], vol. 325, Springer-Verlag, Berlin, 2016.

[4] U. S. Fjordholm, High-order accurate entropy stable numerical schemes for hyperbolic conservation laws, Ph.D. thesis, ETH Zurich, 2013, No. 21025.

[5] Stability properties of the ENO method, Handbook of Numerical Methods for Hyperbolic Problems: Basic and Fundamental Issues, Handbook of Numerical Analysis, vol. 17, Elsevier, 2016, pp. 123-145.

[6] U. S. Fjordholm and S. H. Zakerzadeh, High-order accurate, fully discrete entropy stable schemes for scalar conservation laws, IMA J. Numer. Anal. 36 (2016), no. 2, 633-654.

[7] U.S. Fjordholm, S. Mishra, and E. Tadmor, Arbitrarily high-order accurate entropy stable essentially nonoscillatory schemes for systems of conservation laws, SIAM J. Numer. Anal. 50 (2012), no. 2, 544-573.

[8] — ENO reconstruction and ENO interpolation are stable, Found. Comput. Math. 13 (2013), no. 2, 139-159.

[9] E. Godlewski and P.A. Raviart, Hyperbolic systems of conservation laws, Mathématiques \& Applications (Paris) [Mathematics and Applications], vol. 3/4, Ellipses, Paris, 1991.

[10] A. Harten, J.M. Hyman, and P.D. Lax, On finite-difference approximations and entropy conditions for shocks, Comm. Pure Appl. Math. 29 (1976), no. 3, 297-322, With an appendix by B. Keyfitz.

[11] A. Harten, S. Osher, B. Engquist, and S.R. Chakravarthy, Some results on uniformly high-order accurate essentially nonoscillatory schemes, Appl. Numer. Math. 2 (1986), no. 3-5, 347-377.

[12] H. Holden and N. H. Risebro, Front Tracking for Hyperbolic Conservation Laws, second edition ed., Springer-Verlag Berlin Heidelberg, 2015.

[13] Vladimir Jovanović and Christian Rohde, Error estimates for finite volume approximations of classical solutions for nonlinear systems of hyperbolic balance laws, SIAM J. Numer. Anal. 43 (2006), no. 6, 2423-2449.

[14] S.N. Kruzkov, First order quasilinear equations in several independent variables, Math USSR SB 10 (1970), no. 2, $217-243$.

[15] P. G. Lefloch, J. M. Mercier, and C. Rohde, Fully discrete, entropy conservative schemes of arbitrary order, SIAM J. Numer. Anal. 40 (2002), no. 5, 1968-1992.

[16] R.J. LeVeque, Finite volume methods for hyperbolic problems, Cambridge Texts in Applied Mathematics, Cambridge University Press, Cambridge, 2002.

[17] S. Osher, Riemann solvers, the entropy condition, and difference approximations, SIAM J. Numer. Anal. 21 (1984), no. 2, 217-235.

[18] E. Y. Panov, Existence and strong pre-compactness properties for entropy solutions of a first-order quasilinear equation with discontinuous flux, Arch. Ration. Mech. Anal. 195 (2010), no. 2, 643-673.

[19] E. Tadmor, Numerical viscosity and the entropy condition for conservative difference schemes, Math. Comp. 43 (1984), no. $168,369-381$.

[20] _ The numerical viscosity of entropy stable schemes for systems of conservation laws. I, Math. Comp. 49 (1987), no. $179,91-103$.

[21] _ Entropy stability theory for difference approximations of nonlinear conservation laws and related time-dependent problems, Acta Numer. 12 (2003), 451-512.

[22] L. Tartar, Compensated compactness and applications to partial differential equations, Nonlinear analysis and mechanics: Heriot-Watt Symposium, Vol. IV, Res. Notes in Math., vol. 39, Pitman, Boston, Mass.-London, 1979, pp. 136-212. 